\title{
Maturação Orbitofrontal, Marcadores Somáticos e Vulnerabilidade Precoce: para uma Hipótese Compreensiva de "Miopia Emocional" na Toxicodependência
}

\author{
Marco Torrado ${ }^{1}$ \\ Sílvia Ouakinin \\ Universidade de Lisboa
}

\begin{abstract}
RESUMO - A hipótese de "miopia emocional" constitui uma reflexão teórica de compreensão da vulnerabilidade psicológica identificada em muitos toxicodependentes. Propõe-se uma cooperação, mas não incorporação, de níveis de conhecimento em torno dos determinantes do neurodesenvolvimento, de perspectivas psicanalíticas e de vinculação e de modelos psicobiológicos das toxicodependências. Salientam-se influências ambientais sobre as mudanças na morfologia cerebral, não apenas o trauma precoce ou a privação de cuidados, mas também as decorrentes de consumos abusivos como cernes de vulnerabilidade. Propõese que a hipótese Damasiana dos marcadores somáticos participe nessa formulação. A parca qualidade das interações precoces pode sustentar o desligamento afetivo progressivo, a hipomaturação do cérebro social, o incremento de um padrão alexitímico e a procura urgente de sensações, todos potenciais propiciadores da busca do prazer nas drogas.
\end{abstract}

Palavras-chave: interações precoces, desenvolvimento emocional, córtex orbitofrontal, alexitimia, toxicodependência

\section{Orbitofrontal Maturation, Somatic Markers and Early Vulnerability: A Comprehensive Hypothesis of "Emotional Myopia" in Drug Addiction}

\begin{abstract}
The "emotional myopia" hypothesis is a theoretical reflection to increase the understanding of the psychological vulnerability showed by many drug addicts Instead of an incorporation, a cooperation is proposed of levels of knowledge on the determinants of the neurodevelopment, psychoanalytical and attachment perspectives and psychobiological models of drug addictions. Environmental inputs that change brain morphology are highlighted, not only early trauma or care deprivation but also others derived from the long-term use of drugs as the core of vulnerability. We propose that Damasio's hypothesis of somatic markers forms part of this theoretical formulation. The low quality of early social interactions may support an increasing emotional disengagement, a poor maturation of the social brain, an increase of alexithymic patterns and noveltyseeking behaviours, all potential triggers for searching for pleasure in drugs.
\end{abstract}

Keywords: early interactions, emotional development, orbitofrontal córtex, alexithymia, drug addiction

Desde há várias décadas que a investigação clínica tem reconhecido no córtex pré-frontal, em particular na região orbitofrontal, um papel primordial nos processos reguladores mnésicos, da emoção e das relações interpessoais. Trata-se de uma região filogeneticamente antiga, e especialmente ampla, no hemisfério direito, que recebe aferências de todas as áreas sensoriais e projeta extensas vias para os centros subcorticais reguladores do afeto e da motivação. Parece de fato constituir-se como centro de integração de múltiplos processos que a ele afluem (Mota-Cardoso, 2001), tais como a informação social, a consciência autobiográfica, a ativação emocional e os processos cognitivos superiores. Constitui verdadeiramente um pilar aferidor dos vários níveis de atividade cerebral e mental, ao qual Luria (1980) se referia como um cerne regulador crítico, cuja maturação se processa precocemente no contexto de vividos relacionais gratificantes, de regulação interpessoal e de vinculação primária. Esse contexto primordial de relação, determinística

1 Endereço para correspondência: Clínica Universitária de Psiquiatria e Psicologia Médica, Faculdade de Medicina da Universidade de Lisboa, Av. Prof. Egas Moniz, Lisboa, Portugal. CEP: 1649-028. E-mail: marcovtorrado@gmail.com da maturação orbitofrontal, parece estar implicado na qualidade das futuras regulações internas, nomeadamente ao nível das funções endócrina e imunitária, mas também ao nível superior e interpessoal, que o indivíduo gradualmente ensaiará na ausência das suas primeiras figuras de vinculação. Postula-se, portanto, que a construção de uma vulnerabilidade ao nível das várias funções elencadas poderá edificar-se cedo, e que o confronto do indivíduo com determinadas variáveis ambientais (com elevado impacto no funcionamento cerebral e psicológico) poderá reforçar essa vulnerabilidade e abrir caminho para fenômenos particulares de doença, nomeadamente de perturbações da regulação emocional.

\section{Regulação cortical, interações sociais precoces e vulnerabilidade emocional}

A partilha de emoções e capacidade reflexiva do cuidador com função materna não só emprestam estados mentais organizadores do psiquismo da criança, na linha do que nos descreve Fonagy (e.g. Fonagy, Gergely \& Target, 2008), como lhe confere uma leitura das emoções experienciadas, pela prévia leitura materna que lhe é devolvida, oferecendo a 
oportunidade de desenvolvimento de um espaço transitivo de experiência que permitirá alcançar mecanismos primordiais de autorregulação, sem depender na totalidade do regulador externo. As protoconversações do primeiro trimestre de vida entre mãe e bebê (mediadas pelo olhar, vocalizações e movimentos das mãos e braços), se dotadas de ressonância intersubjectiva e sincronia afetiva, permitem a maturação da atividade do hemisfério direito pela regulação materna de neurotransmissores e modulação dos níveis de ativação somática do bebê (Schore, 1997; Trevarthen, 1993). Essa regulação externa desencadeia padrões neurohormonais e substâncias neuromoduladoras catecolaminérgicas essenciais à morfogênese das regiões corticais (Hofer, 1990). Ao longo do primeiro ano, essas interações determinam ainda que se processe de forma decisiva a organização e processos regulatórios do sistema límbico do hemisfério direito não-verbal e do cingulado anterior direito, implicados no reconhecimento de faces e processamento não consciente das emoções. Mais tarde, a maturação das regiões orbitofrontais direitas (no final do segundo ano de vida) consubstancia uma evidente dominância do hemisfério direito ao longo dos primeiros três anos de vida, antecedendo um período de maior consolidação à esquerda por meio do início da transmissão pelo corpo caloso e do crescimento dos axônios do setor pré-frontal esquerdo para o direito.

O forte impacto das experiências sociais precoces na maturação cerebral, em especial à direita, foi de certo modo fundamentado por investigações de Chiron et al. (1997), ao demonstrar a dominância do hemisfério direito em crianças ainda em estágios de desenvolvimento pré-verbal. Nessas interações, os modelos internos de funcionamento formados no contexto da relação de vinculação são processados e armazenados em sistemas de memória implícita (processsual, sensorial) no hemisfério direito, consubstanciando o substrato de conteúdos que mais tarde integrarão memórias declarativas e não-declarativas inerentes ao self.

O sistema orbitofrontal está intimamente conectado ao sistema nervoso autônomo e à formação reticular, promotora da ativação (arousal) do organismo. O fato desse sistema ser a única estrutura cortical dotada dessas conexões permite-lhe regular as respostas autonômicas decorrentes de estímulos sociais (Zald \& Kim, 1996), tais como modular o comportamento instintivo (Starkstein \& Robinson, 1997) e promover o controlo inibitório (Garavan, Ross, \& Stein, 1999), expandindo essa ação a todo o hemisfério direito. Estudos da última década (e.g., Barbas, Saha, Rempel-Clover, \& Ghashghaei, 2003) demonstraram a ligação íntima entre regiões frontais, em particular a região orbitofrontal e as estruturas autonômicas (por meio de sinapses entre axônios pré-frontais e estruturas autonômicas via hipotálamo), sendo salientadas as conexões robustas bilaterais dessa região com a amígdala o que, concomitantemente, a torna numa estrutura muito influente no âmbito da expressão emocional. Barbas et al. (2003) realçam que lesões nessa região são compatíveis com uma expressão afetiva pobre ou desapropriada, assim como perturbações da componente emocional intrínseca às tomadas de decisão. A atividade desse sistema é, portanto, crítica para a modulação dos comportamentos socioemocionais e para a regulação homeostática dos estados corporais e motivacionais, e cujo envolvimento nos processos de vinculação é central.

A região orbitofrontal encontra-se especialmente expandida no hemisfério direito, área onde predominantemente estão alocados os processos automáticos a partir dos quais é processada a relevância afetiva dos estímulos externos e atribuída uma valência positiva ou negativa, em função do nível de prazer ou desprazer. Adicionalmente, abarca aquilo que Schore (2001) denomina de léxico afetivo não-verbal, um vocabulário para os sinais afetivos não verbais tais como expressões faciais, gestos e prosódia (Bowers, Bauer, \& Heilman, 1993). Nesse sentido, o hemisfério direito é mais rápido do que o esquerdo em processos automáticos de avaliação preliminar da valência de expressões faciais de emoções (Pizzagalli, Regard, \& Lehmann, 1999).

A atividade orbitofrontal parece participar na capacidade reflexiva, corolário daquilo que, na óptica de Schore (2001), resulta de processos de vinculação segura e de sintonia afetiva com os cuidadores primordiais, permitindo ao indivíduo compreender os seus estados mentais e depreender os dos outros. Segundo Fonagy e Target (1997) e Fonagy et al. (2008), essa função reflexiva é desenvolvida, em um primeiro plano, na relação com um cuidador dotado dessa mesma função, envolvendo o hemisfério direito. Trata-se de uma operação mental que incrementa a percepção de estados emocionais de outros e, portanto, que oferece os precursores da capacidade empática. Essa função, teoricamente próxima da capacidade de inferir sobre os estados mentais dos outros (Teoria da Mente) e de níveis mais diferenciados de consciência emocional (Subic-Wrana, Beutel, Garfield, \& Lane, 2011), eleva o potencial adaptativo dos indivíduos em termos de funcionamento social. Estudos desenvolvidos por Stone, Baron-Cohen e Knight (1998) demonstraram que indivíduos com lesões bilaterais orbitofrontais demonstravam resultados pobres (típicos de indivíduos com perturbações do espectro autista) em tarefas requerentes de um raciocínio social mais subtil, contrariamente ao sucedido com sujeitos com outro tipo de lesões (e.g., região dorsolateral do córtex), sugerindo que o córtex orbitofrontal está particularmente envolvido em tarefas com componente afetivo e que exigem uma Teoria da Mente.

Schore (1994, 1997, 2000) salienta que as experiências relacionais precoces pautadas por episódios de maus-tratos/ abuso, negligência e trauma promovem microalterações neuronais que poderão se tornar críticas para o desenvolvimento saudável das áreas corticolímbicas. Sublinha que a morte celular, em períodos críticos de neurodesenvolvimento, nomeadamente de neurônios dos córtices orbitofrontal e ou temporal especialmente implicados na resposta a estímulos faciais, leva a défices permanentes na leitura de expressões faciais de estados emocionais. Estudos da década de 80 trouxeram evidência de défices de descodificação emocional em crianças maltratadas (e.g., Camras, Grow, \& Ribordy, 1983). Dado que as áreas orbitofrontais estão intimamente ligadas aos circuitos límbicos e às enervações do sistema nervoso autônomo, um desenvolvimento parcelar desses circuitos, sustentado em variáveis ambientais de tipo relacional rejeitante/desorganizado, poderá resultar em um contexto cortical frontolímbico pobre com o qual os componentes simpático e parassimpático não poderão 
operar reciprocamente (Bernston, Cacioppo, \& Quigley, 1991). Esse tipo de organização do controle autonômico previne a integração de estados autonômicos mais primitivos, o que seria fundamental à elaboração de novos estados psicofisiológicos mais diferenciados. Essa fragilidade nas capacidades regulatórias, mesmo sobre situações de stress moderado, torna o equipamento cerebral vulnerável à desorganização e a mudanças no desenvolvimento afetivo pautadas por padrões descontínuos e lábeis. Partindo dessa ordem de assumpções, De Brito et al. (2013) mostraram que um conjunto de crianças e adolescentes sujeitos a práticas de maus-tratos e negligência, comparadas a controles emparelhados em diversas variáveis demográficas e psicopatológicas, apresentavam um volume de substância cinzenta menor ao nível do córtex orbitofrontal, da região medial e do giro temporal médio. Sendo os padrões disfuncionais dessas regiões altamente correlacionados com perturbações executivas, nomeadamente na tomada de decisão, mas também da regulação emocional (por sua vez, compatíveis com várias perturbações psiquiátricas), os autores sugerem que esse défice poderá representar um fator de vulnerabilidade neural latente para a emergência posterior de psicopatologia nos indivíduos cujas fases precoces do desenvolvimento foram marcadas por padrões relacionais disfuncionais.

\section{Desenvolvimento afetivo, psicopatologia e marcadores somáticos}

O conhecimento neurocientífico na última década tem reforçado a evidência da presença de perturbações no funcionamento do córtex orbitofrontal em diferentes psicopatologias. Helen Barbas focou as características neurobiológicas particulares dessa área cerebral como relevantes na compreensão da sua vulnerabilidade específica às perturbações psiquiátricas, descrevendo o envolvimento particular da região posterior do córtex orbitofrontal, por meio de robustas projeções com a amígdala, no processamento sequencial das emoções e na expressão emocional desregulada em perturbações psiquiátricas, tais como perturbações da ansiedade (Barbas, 1995; Barbas, Zikopoulos, \& Timbie, 2011; Remper-Clower, 2007).

No domínio das interações sociais, Kolb, Pellis e Robinson (2004) e Robinson e Kolb (1999) têm demonstrado a implicação determinante do córtex orbitofrontal de ratos (região análoga às áreas orbitais dos primatas) no desenvolvimento e manutenção dessas interações, por meio de estudos com roedores lesionados nessas regiões e de outros sobre administração de opiáceos. Os resultados desses estudos mostraram que os ratos, de quaisquer idades, com lesões orbitofrontais evidenciavam défices crônicos no seu comportamento social, não modificáveis ao longo do desenvolvimento. No caso particular das lesões neonatais em ratos jovens, embora as funções orbitofrontais de domínio cognitivo e motor pudessem ser compensadas pelo funcionamento de outras regiões (e.g., ventromedial do córtex) em virtude de mecanismos de neuroplasticidade, essa compensação não era possível relativamente à recuperação do normal comportamento social. De modo similar, os autores mostraram que a administração de morfina em ratos produzia um decréscimo da densidade dendrítica espinal nas células piramidais do córtex pré-frontal ventromedial, mas, inversamente, produzia um aumento na densidade espinal e de cerca de 30\% no número de sinapses no córtex orbitofrontal, conferindo evidência à alteração da organização sináptica produzida pela administração de substâncias, com impacto no funcionamento dessas regiões cerebrais. De Drevets, Gadde e Krishman (1999) tinham anteriormente salientado que o fluxo sanguíneo no cérebro se encontra alterado nas regiões pré-frontais em certas perturbações do humor, fato compatível com as alterações descritas na literatura a propósito do consumo de substâncias.

Esses estudos sugerem, na ótica de Kolb et al. (2004), que as alterações no nível orbitofrontal, quer por perturbações no desenvolvimento, quer por alterações produzidas por administração de substâncias, podem contribuir para dificuldades na organização do comportamento social e afiliativo, podendo essas alterações assumir-se como potencial fator de vulnerabilidade para a emergência de perturbações relacionais eventualmente manifestadas sob a forma de condições psicopatológicas.

Resultados obtidos pela equipe de Damásio, especificamente por Bechara, H. Damasio e A. R. Damasio (2000), sugeriram que as áreas do córtex pré-frontal, nomeadamente as regiões orbitofrontal e ventromedial, promovem influências ou enviezamentos não conscientes sobre o comportamento, prévios ao conhecimento consciente. Tais regiões são consideradas estruturas centrais na tomada de decisão, mediando a influência dos aspectos emocionais nas decisões por meio de processos a que Damásio denominou de marcadores somáticos (Damásio, 1994/1999). Numa perspectiva similar, Dolan (1999) sustentou que a região orbitofrontal detinha um papel relevante na codificação do significado afetivo das opções comportamentais futuras, permitindo o contato entre a informação emocional armazenada e processos de seleção da ação. De outro modo, essas regiões parecem assumir-se como correlato cerebral de configurações mentais (componentes cognitivos e afetivos) que guiam a avaliação da experiência e, concomitantemente, as ações futuras, o que, na ótica de Schore (1997, 2000), detém um paralelismo relativamente evidente com a asserção dos modelos internos dinâmicos de funcionamento propostos por Bowlby (1981), ou das representações mentais (Main, Kaplan, \& Cassidy, 1985), ambos desenhados em ampla medida no contexto das interações sociais precoces de vinculação.

Os marcadores somáticos compõem um fenômeno neurobiológico particular do uso de sentimentos criados a partir de emoções secundárias, em que ambos se associaram por via da aprendizagem de certo tipo de resultados futuros ligados a certo tipo de acontecimentos. Quando um marcadorsomático negativo é associado a um dado resultado futuro, a combinação resulta em um sinal de alarme para o organismo. Em contrário, quando positivo, a combinação surge como um incentivo. Podem, portanto, ser considerados como mecanismos de avaliação automática das consequências previsíveis de dados acontecimentos (Damásio, 1994/1999).

Esses marcadores são processos de influência, não determinísticos, sendo que a constituição de marcadores 
somáticos adaptativos requer a condição de uma certa "normalidade" cerebral e cultural. Nesse contexto, e como nos recorda Damásio (1994/1999), a maior parte dos marcadores somáticos que usamos para a tomada racional de decisões é provavelmente desenvolvida a nível central durante os processos de educação e socialização, por meio da associação de categorias específicas de estímulos a categorias específicas de estados somáticos. Estes processos envolvem regiões frontais e pré-frontais do cérebro.

De acordo com Damásio (1994/1999), os marcadores somáticos são, portanto, adquiridos por meio da experiência, sob o controle de um sistema interno de preferências que é influenciado pelas circunstâncias externas que envolvem o indivíduo e com as quais este interage, nomeadamente fenômenos, objectos, ambiente, aspectos éticos e normas sociais. O sistema interno de preferências, orientado para a sobrevivência e posicionando-se, portanto, em um sentido motivacional de evitamento da dor e da procura do prazer, é determinado desde o início do desenvolvimento individual por contato com múltiplos eventos externos. Nos tempos precoces de desenvolvimento, são os pais que normalmente objectivam a ética e as convenções sociais estabelecidas pela cultura envolvente, aplicando recompensas e castigos cujo contato com o sistema motivacional faz aumentar o reportório de estímulos marcados automatica e somaticamente. $\mathrm{O}$ autor enfatiza que "o conjunto crítico e formativo de estímulos para os emparelhamentos somáticos é, sem dúvida, adquirido na infância e na adolescência." (p. 191).

Em termos neurais, os marcadores somáticos dependem dos processos de aprendizagem que associem determinados tipos de fenômenos à produção de estados corpóreos de valência positiva ou negativa, sendo que tal associação entre o tipo de estado somático e de sentimento produzido em um dado indivíduo é determinado em função do seu contexto situacional e da sua história pessoal. As regiões pré-frontais abarcam o sistema neural por excelência envolvido na recepção dos sinais enviados por todas as partes do corpo, inclusivamente de vários setores biorreguladores como o tronco cerebral, a amígdala ou o hipotálamo e, como tal, constitui uma região cerebral crítica para a aquisição de sinais pelos marcadores somáticos. Por outro lado, as suas redes neuronais estabelecem representações de disposições para certas associações entre eventos ou objectos, em função da experiência individual e da relevância pessoal atribuída, tornando a categorização da experiência em um processo muito pessoal e único, diferente de pessoa para pessoa e, portanto, contingente (Bechara et al., 2000; Damásio, 1994/1999).

Esses marcadores somaticamente estabelecidos poderão atuar de modo oculto e sem percepção consciente, como já foi dito, enviando sinais de luta/fuga ao organismo por meio de estados corporais (por exemplo, dolorosos), no sentido de evitar que o indivíduo tome uma determinada opção (ou similar) que no passado tenha conduzido a um resultado pouco adaptativo e que, por sua vez, tenha promovido esse mesmo marcador somático. Esses marcadores, que funcionam de forma automática e sem passagem pela consciência, utilizam circuitos emocionais denominados "como se". A emergência desses mecanismos decorre do próprio desenvolvimento individual, resultando da categorização de situações repetidas e de um progressivo abdicar dos estados somáticos. Esses, sendo consolidados positiva ou negativamente durante a infância e adolescência, eram nesse período os determinantes quase exclusivos dos processos de tomada de decisão.

Essa gradual autonomia conseguida pelos mecanismos "como se" sucede, em parte, graças à possibilidade de simbolização dos estados somáticos. Ainda assim, Damásio (1994/1999) postula que, mesmo em um contexto de maior diferenciação emocional, os sentimentos não passam a ser todos "como se", pois desse modo o indivíduo não acederia a qualquer noção de modulação afetiva em permanente mudança, o que aliás constitui um traço evidente do nosso funcionamento mental. Algumas pessoas utilizam de forma relevante esses processos "como se", denunciando um funcionamento mais diferenciado. Para elas, as decisões são recorrentemente tomadas sem o envolvimento da atenção sobre os sinais de um estado corporal ou seu substituto ("como se"). Outras pessoas, no entanto, possivelmente dependem em muito da percepção dos estados somáticos, na ausência de capacidades de simbolização das valências e ativações emocionais subjacentes a esses estados, o que poderá ser compatível com processos emocionais deficitários na consciência, identificação e expressão emocionais.

\section{Marcadores somáticos e toxicodependência: uma hipótese de vulnerabilidade psicossomática.}

Na procura de um modelo explicativo dos défices de processamento cognitivo-afetivo presentes em muitos toxicodependentes, e fortemente influenciados pelo modelo de Damásio, Verdejo-Garcia e Bechara (2009) propuseram aquilo a que denominaram de modelo dos marcadores somáticos da adição. Considerando que os processos de tomada de decisão dependem, em diferentes níveis, dos substratos neurais que regulam a homeostase e os processos emocionais, esses autores salientaram que nos indivíduos com dependência de substâncias parecem estar envolvidas fragilidades nos componentes centrais dos circuitos envolvidos na ativação de estados somáticos e nas tomadas de decisão. De acordo com esse modelo, na toxicodependência, o indivíduo torna-se incapaz de desenvolver escolhas que impliquem resultados não imediatos e de reflectir sobre as consequências a longo prazo das suas decisões. As escolhas feitas com base em resultados a longo prazo, diferentes daquelas que implicam resultados imediatos, requerem que os marcadores somáticos ativados por pensamentos acerca das consequências negativas subjacentes à procura e consumo de drogas sejam dominantes sobre os sinais impulsionados pelo reforço imediato do consumo. Baseados nesse modelo, Verdejo-Garcia e Bechara (2009) sugerem dois tipos de condições globais que podem estar envolvidas no processo deficitário de tomada de decisão e na competência reduzida desses indivíduos em resistir ao desejo do consumo: (1) uma das condições parece envolver uma disfunção dos sistemas orbitofrontal e ventromedial do córtex pré-frontal, fundamentais no processamento dos estados emocionais somáticos induzidos pelos ativadores (triggers) secundários do consumo (e.g., recordação do uso de uma substância e dos efeitos associados). O sistema reflexivo, correlativo ao 
normal funcionamento dessas regiões cerebrais, perdeu, na perspectiva desse modelo, a sua capacidade para processar e ativar sinais somáticos associados a planos futuros, como por exemplo, as consequências negativas inerentes aos consumos; (2) a outra condição poderá envolver uma hiperativação da amígdala (estrutura essencial ao processamento dos estados emocionais somáticos decorrentes de ativadores primários do consumo de drogas). A atividade desse sistema impulsivo tornou-se alterada de tal forma que diminui o impacto emocional/somático dos estímulos naturais, mas exacerba o impacto somático dos planos para a obtenção imediata de drogas. Desse modo, os ativadores associados à obtenção de drogas adquirem propriedades de ativação de processos bottom-up que envolvem estados somáticos involuntários, através da amígdala. Esse enviezamento somático de bottomup poderá modular mecanismos cognitivos de top-down (nos quais as áreas pré-frontais são substratos fundamentais), sendo que, revelando-se suficientemente forte, poderá interferir significativamente com os mecanismos top-down necessários ao normal funcionamento do sistema reflexivo, o que é determinante em termos do autocontrole necessário à decisão de não consumir.

Esse modelo é largamente conciliável com a observação clínica de que muitos indivíduos com dependência de substâncias parecem deter fragilidades importantes nos juízos avaliativos das tomadas de decisão, com os referidos correlatos na área cortico-subcortical. Esses défices não se observam apenas no domínio do controle do impulso para consumir face a ativadores primários ou secundários, como também na capacidade para antecipar o legado de efeitos nocivos decorrentes dessa ação e de avaliá-los como dominantes sobre a recompensa imediata dos efeitos da substância, tornando os estímulos naturais, e promotores de contentamento na nossa matriz sociológica, elementos inferiores na economia psicológica do indivíduo (Damásio, 1994/1999; Félix da Costa, 1995; Verdejo-Garcia \& Bechara, 2009;). Essa perspectiva, sustentada na evidência e explicação dos défices executivos de planejamento e de controle inibitório face à possibilidade de recaída, não parece, contudo, excluir a hipótese compreensiva de uma vulnerabilidade biopsicossocial prévia ao percurso de toxicodependência, o que não justifica um discurso defensor da presença de nosologias psicopatológicas particulares na gênese da própria toxicodependência.

Sem eliminar a conjectura de que em uma parte substancial dos indivíduos toxicodependentes a sua problemática é explicável predominantemente em um nível psicobiológico determinado por consumos continuados, é aqui hipotetizado que, numa outra parte, a disfunção orbitofrontal possa deter a sua origem não exclusivamente no percurso de consumos, mas também em uma vulnerabilidade incrementada no percurso do seu desenvolvimento afetivo. Essa perturbação decorreria primordialmente de uma (sub)maturação frontal precoce, construída no seio de cuidados insatisfatórios na esfera afectivo-emocional e, posteriormente, reforçada por contextos de ordem social/ambiental pouco promotores da simbolização, logo, robustecendo uma certa dependência da leitura somática como determinante da ação.

Essa hipótese teórica sustenta-se na observação, em contexto clínico, dos relatos de muitos toxicodependentes cujos relatos sobre sua história pregressa parecem incorporar tipologias particulares de representações subjacentes às interações precoces, no contexto das primeiras etapas do seu desenvolvimento. É relativamente comum reconhecer, nesses pacientes, relatos retrospectivos de negligência, maus-tratos e rejeição. Esses relatos, envolvendo conteúdos de vivências muito prematuras subsidiam representações mentais das figuras parentais normalmente envoltas de parco suporte afetivo, desamparo e, por vezes, de modo (aparentemente) inverso ou adicional, de tentativas de controle exacerbado ou intrusão face a uma crescente autonomia comportamental, em particular na adolescência. Tomando como referenciais globais as perspectivas psicanalíticas e da vinculação, em um contexto de práticas relacionais de cuidados, é lícito considerar que um indivíduo em desenvolvimento que não beneficia (ou percepciona) uma responsividade afetiva marcada pelo envolvimento, calor e aprovação emocionais, tenderá gradualmente a não estabelecer vínculos tomados como securizantes, porquanto os mesmos nem seriam adaptativos ou potenciadores da sobrevivência desse indivíduo. Em um paralelismo com a hipótese damasiana dos marcadores somáticos, hipotetiza-se, aqui, que esses padrões comportamentais pouco gratificantes, perpetrados pelos cuidadores, possam impulsionar, na criança, o emparelhamento de estados somáticos a sentimentos de rejeição vividos em contexto relacional, fazendo emergir marcadores somáticos negativos justapostos a resultados futuros de fraca responsividade (ou, de outro modo, de precária sintonia emocional). Estes poderão conduzir o indivíduo a uma recusa gradativa do rumo de ação negativo (leia-se, um progressivo abdicar de investimento relacional) de forma a proteger-se de prejuízos relacionais futuros.

Essa assintonia afetiva em desenvolvimento, indício de desinvestimento objectal ou mesmo desligamento por parte do indivíduo em crescimento, não só comporta implicações relativamente directas na qualidade dos modelos internos de funcionamento, como pode promover aquilo a que Farate (2001) denominou, no contexto da adolescência, de situações de "risco relacional". Na perspectiva de Farate, a percepção negativa da atitude relacional das figuras parentais influi negativamente na qualidade da interação com o meio, predispondo o indivíduo em desenvolvimento à procura de atividades ou contextos que não solicitem uma verdadeira ligação afetiva. Mais recentemente, Hawkley, Preacher e Cacioppo (2007) afirmaram que indivíduos que se sentiram em maior "isolamento" relacional percepcionam as interacções sociais positivas como menos gratificantes e prazenteiras do que indivíduos que se sentem ligados afectivamente a outros, sustentando que o desapego relacional pode gradativamente dar suporte a vias alternativas da procura do prazer, tão imediatas quanto possível se o nível de experiência emocional se pautar sobretudo por modalidades de funcionamento sensório-motor.

Porque a maturação pré-frontal é em muito determinada pela responsividade precoce materna (que se pretende suficientemente boa, à luz da perspectiva de Winnicott, 1971) e coadjuvada por figuras que sequencialmente prolongam a função reflexiva do primeiro cuidador (i.e. a figura paterna), a situação hipotética aqui descrita, embora não testada, acarretaria uma fragilização dessa maturação, oferecendo 
o substrato neural para um funcionamento particularmente centrado nos estados somáticos e parco de simbolização. Face à rarefacção de circuitos emocionais "como se", que substituíssem um processamento exclusivamente somático, o funcionamento de indivíduos com esta hipomaturação fica pautado por uma sensorialidade e uma certa dependência dos estados corpóreos enquanto recurso possível da leitura do emocional, o que é compatível com a hipótese de uma vulnerabilidade alexitímica. Esse défice de processamento afetivo, por sub-maturação da região orbitofrontal e possível hiperatividade da amígdala, torna, nessa elaboração teórica esses indivíduos, ao longo dos primeiros estágios do desenvolvimento, particularmente mais focados na procura de sensações (prazer) e dotados de um estilo cognitivo mais externalizado e menos elaborado. Essa conjunção de aspectos fornece o terreno neural e, concomitantemente, experiencial para o desenvolvimento de uma certa "miopia para o futuro", à qual Damásio (1994/1999) se refere como uma metáfora do funcionamento neuropsicológico de muitos toxicodependentes. Este autor sugere que alguns indivíduos com dependências de drogas detêm um paralelismo singular com pacientes com lesões frontais, pela similitude na perturbação de planejamento e de antecipação de resultados futuros em virtude da disfunção dos córtices ventromedial e orbitofrontal inerente à carreira de consumos. Na linha de Damásio, e de acordo com a hipótese aqui formulada, sugere-se o termo "miopia emocional" para caracterizar um fenômeno de gênese biorrelacional e de expressão somatopsíquica, presente em alguns indivíduos em desenvolvimento, caracterizado por um défice cognitivo-experiencial e de simbolização estabelecido por constrangimentos nas interações sociais precoces. Estas, quando pouco gratificantes, poderão ser indutoras de uma sub-regulação cortico-subcortical, sustentando um funcionamento pautado pelo concreto, de parca diferenciação na organização da experiência emocional e centrado nos estados somáticos. Tal conjunto de características de funcionamento, conciliável com o desenvolvimento do traço alexitímico, é igualmente próximo do termo "cegueira emocional" proposto por Lane, Kaszniak, Ahern e Schwartz (1997) ao se referirem a pacientes com maiores dificuldades em catalogar e analisar estados emocionais. $O$ fenômeno aqui proposto pode sustentar um eventual fator de vulnerabilidade para o desenvolvimento de perturbações psicopatológicas, nomeadamente associadas ao abuso de substâncias, dada a necessidade dos indivíduos em promover uma ativação do sistema apetitivo (a procura do prazer) por meio de recursos com efeitos diretos sobre os estados somáticos, em detrimento da sua obtenção por meio de ações mais elaboradas (e.g., o prazer afiliativo) cujo reforço não só não é imediato como também pressupõe um nível de experiência emocional mais diferenciado.

A perspectiva psicanalítica de Farate (2001) sobre o "risco relacional", interpretada como descritiva de um fator particular de vulnerabilidade em jovens no despertar para o consumo abusivo de substâncias, e de certo modo uma forma de mediar uma fragilidade interna crescente (coping), detém alguma similaridade com a hipótese de miopia emocional. Esta hipótese assume uma perspectiva predominantemente psicossomática para caracterizar um funcionamento edificado em ambientes afetivo-relacionais tendencialmente frustrantes, promotores de vínculos inseguros e concomitantes autopercepções negativas, cuja consequência neurodesenvolvimental pode "empurrar" o indivíduo para um funcionamento centrado em uma leitura prioritariamente sensorial da sua vida emocional. Tal como Farate refere, a conduta do consumo de substâncias pode ter lugar na gestão de um alargado desconforto emocional vivido de modo pouco diferenciado, não catalogado (simbolizado) ou expressado no domínio psicoafetivo.

A investigação tem apoiado, de forma mais ou menos evidente e com impacto no domínio preventivo, a relevância do suporte emocional precoce e prolongado pela adolescência como fator central em uma perspectiva de proteção/risco face ao abuso de substâncias (e.g. Farate, 2001). Tem sido demonstrado que aqueles que relatam relações saudáveis com os pais, pautadas por padrões comunicacionais francos, são adolescentes que tendencialmente se envolvem menos em práticas de uso regular de substâncias (Stronski, Ireland, Michaud, Narring, \& Resnick, 2000). Um contexto familiar caloroso e com suporte afetivo, caracterizado por ligações sentidas como gratificantes e níveis baixos de conflito intrafamiliar, tem sido caracterizado como um fator preditor de um menor risco de início de consumos de drogas ilícitas na adolescência. Fatores como a supervisão parental próxima e o estabelecimento de uma comunicação clara com crianças sobre normas de comportamento também se relacionam com menor risco de consumo de drogas. Por outro lado, a participação em atividades de grupo com características antissociais parece predizer um maior risco de uso de drogas ilícitas, o que se inverte face a uma maior participação em grupos proativos (EMCDDA, 2009; Guo, Hill, Hawkins, Catalano, \& Abbott, 2002).

\section{Considerações finais}

O modelo de miopia emocional constitui uma hipótese teórica de compreensão da vulnerabilidade psicossomática identificada em muitos indivíduos com perturbações associadas ao uso e abuso de substâncias, baseada no conhecimento e reflexões clínicas e no legado que as perspectivas neurocientíficas têm promovido para a compreensão dos fenômenos mentais. É uma tentativa de estabelecer de uma forma cooperativa, mas não incorporativa, associações entre níveis de conhecimento em torno dos determinantes relacionais do neurodesenvolvimento, das perspectivas clínicas analíticas e da vinculação e modelos psicobiológicos explicativos das toxicodependências. Essa hipótese salienta as influências ambientais sobre as mudanças na morfologia cerebral, não apenas o trauma precoce ou a privação de cuidados e suporte afetivo, mas também aquelas que derivam de consumos continuados de certas substâncias, de modo que, em um largo espectro de situações, as primeiras poderão auxiliar as segundas. A extrapolação dos achados de Damásio para o domínio das ciências afetivas, pela consideração de marcadores somáticos do impacto das interações precoces em um progressivo desligamento afetivo, possibilita teorizar que a fraca maturação de regiões cerebrais implicadas no prazer social e afiliativo, um funcionamento 
alexitímico e uma procura de sensações no imediato seriam fatores precipitantes para o recurso ao prazer imediato do consumo. Contudo, o entendimento dos mecanismos neurobiológicos subjacentes aos vários processos analisados (vinculação precoce, emoção, dependência química) não oferece, nessa perspectiva, um caminho para o entendimento da problemática em um contexto de pleno determinismo biológico, envidando, pelo contrário, pontes para uma tentativa de compreensibilidade de vários fatores que podem concorrer para o desenvolvimento psicológico e psicopatológico individual.

Esta reflexão teórica não assume, por isso, um caráter explicativo e compreensivo de todos os processos de toxicodependência, ou da globalidade dos percursos de mau uso de substâncias. Consequentemente, a hipótese de miopia emocional não parece ser controversa com as opções individuais de inúmeras pessoas que, seja pela curiosidade ou pelo convicto desejo de indagar áreas menos desvendadas do seu funcionamento mental, utilizam substâncias de forma relativamente lúdica e ocasional (no contexto da sua liberdade individual) em ambientes recreativos, não menos integradas nos códigos sociais que delimitam a "normalidade" na nossa cultura. Ainda assim, tais condutas pressupõem a manutenção do controlo inibitório (ao nível neural e comportamental) e de uma capacidade sólida de planejamento e antecipação dos efeitos futuros de tais tomadas de decisão. Sugere-se, contudo, que indivíduos que padeçam de miopia emocional talvez tenham maior dificuldade em planejar e antecipar consequências. Isso porque suas características neurobiológicas e psicológicas influenciam a partida o propósito dos primeiros consumos, que, nesses casos, poderá remeter mais para uma procura implícita de sensações ativadoras e de valência positiva, na ausência da antecipação das consequências em virtude de défices de processamento cognitivo-afetivo prévios. $\mathrm{O}$ impacto nas vias de reforço pode ser de tal modo potente que os défices experienciais afetivos, assim como as alterações cerebrais, tenderão a consolidar-se em função dos consumos e de uma frequente escalada de desinvestimentos pessoais, sociais e afiliativos.

Khantzian $(1985,1990)$ enfatiza a importância da experiência psicoterapêutica com esses pacientes como promotora de uma nova experiência relacional (e diria afiliativa) que permita a aquisição progressiva de funções reguladoras da tensão interna por meio de uma aceitação quase incondicional, sem negligenciar, contudo, uma atitude simultaneamente afirmativa. Acrescenta-se a possibilidade do contexto de tratamento poder oferecer novos símbolos e significados para a experiência. Os autores do presente trabalho retomam a ideia de que são as experiências com os objetos que são internalizadas, e não os objetos per se, daí a possibilidade da sua reparação em novas experiências com novos objetos de relação, em um labor progressivo e tolerante.

\section{Referências}

Barbas, H. (1995). Anatomic basis of cognitive-emotional interactions in the primate prefrontal cortex. Neuroscience \& Biobehavioral Reviews 19, 499-510.

Barbas, H., Saha, S., Rempel-Clower, N., \& Ghashghaei, T. (2003). Serial pathways from primate prefrontal cortex to autonomic areas may influence emotional expression. BMC Neuroscience, $4,25$.

Barbas, H., Zikopoulos, B., \& Timbie, C. (2011). Sensory pathways and emotional context for action in primate prefrontal cortex. Biological Psychiatry, 69, 1133-1139.

Bechara, A, Damasio, H., \& Damasio, A. R. (2000). Emotion, decision making and the orbitofrontal cortex. Cerebral Cortex, 10, 295-307.

Bernston, G. G., Cacioppo, J., \& Quigley, K. S. (1991). Autonomic determinism: The modes of autonomic control, the doctrine of autonomic space, and the laws of autonomic constraint. Psychological Review, 98, 459-487.

Bowers, D., Bauer, R. M. \& Heilman, K. M. (1993) The nonverbal affect lexicon: theoretical perspectives from neuropsychological studies of affect perception. Neuropsychology, 7, 433- 444.

Bowlby, J. (1981). Attachment and loss (Vol. III). Loss: sadness and depression. New York: Basic Books.

Camras, L., Grow, G., \& Ribordy, S. (1983). Recognition of emotional expression by abused children. Journal of Clinical Child Psychology, 12, 325-328.

Chiron, C., Jambaque, I., Nabbout, R., Lounes, R., Syrota, A., \& Dulac, O. (1997). The right brain hemisphere is dominant in human infants. Brain, 120, 1057-1065.

Damásio, A. (1999). O erro de Descartes: emoção, razão e cérebro humano (19 ed.). Mem Martins, Portugal: Publicações EuropaAmérica (Trabalho original publicado em 1994)

De Brito, S. A., Viding, E., Sebastian, C. L., Kelly, P. A., Mechelli, A., Maris,H., \& McCrory, E. J. (2013). Reduced orbitofrontal and temporal grey matter in a community sample of maltreated children. Journal of Child Psychology and Psychiatry, 54, 105-112.

Drevets, W. C., Gadde, K. M., \& Krishman, K. R. R. (1999). Neuroimaging studies of mood disorders. In D. S. Charney, E. J. Nestler \& B. S. Bunney (Eds.), Neurobiology of mental illness (pp 394-418). New York: Oxford University Press.

Dolan, R. J. (1999). On the neurology of morals. Nature Neuroscience, 2, 927-929.

European Monitoring Centre for Drugs and Drug Addiction [EMCDDA] (2009). Preventing later substance use disorders in at-risk children and adolescents: A review of the theory and evidence base of indicated prevention. Luxembourg: EMCDDA.

Farate, C. (2001). O acto de consumo e o gesto que consome-Risco relacional e consumo de drogas no início da adolescência. Coimbra: Quarteto.

Félix da Costa, N. (1995). Contentamento e prazer. Toxicodependências, 1, 5-13.

Fonagy, P., Gergely, G., \& Target, M. (2008). Psychoanalytic constructs and attachment theory and research. In J. Cassidy \& P. Shaver (Eds.), Handbook of attachment: Theory, research, and clinical applications (pp. 783-810). New York: The Guilford Press. 
Fonagy, P., \& Target, M. (1997). Attachment and reflective function: Their role in self-organization. Development and Psychopathology, 9, 679-700.

Garavan, H., Ross, T. J., \& Stein, E. A. (1999). Right hemisphere dominance of inhibitory control: An event-related functional MRI study. Proceedings of the National Academy of Sciences of the United States of America, 96, 8301-8306.

Guo, J., Hill, K. G., Hawkins, J. D., Catalano, R. F., \& Abbott, R. D. (2002). A developmental analysis of sociodemographic, family, and peer effects on adolescent illicit drug initiation. Journal of the American Academy of Child and Adolescent Psychiatry, 41, 838-845.

Hawkley, L. C., Preacher, K. J., \& Cacioppo, J. T. (2007). Multilevel modeling of social interactions and mood in lonely and socially connected individuals: The MacArthur social neuroscience studies. In A. D. Ong \& M. van Dulmen (Eds.), Handbook of methods in positive psychology (pp. 559-575). New York: Oxford University Press.

Hofer, M. A. (1990). Early symbiotic processes: Hard evidence from a soft place. In R. A. Glick \& S. Bone (Eds.), Pleasure beyond the pleasure principle (pp. 55-78). New Haven: Yale University Press.

Khantzian, E. J. (1985). The self-medication hypothesis of addictive disorders: Focus on heroin and cocaine dependence. American Journal of Psychiatry, 142, 1259-1264.

Khantzian, E. J. (1990). Self-regulation and self-medication factors in alcoholism and the addictions. Similarities and differences. Recent Developments in Alcoholism, 8, 255-271.

Kolb, B., Pellis, S., \& Robinson, T. E. (2004). Plasticity and functions of the orbital frontal cortex. Brain and Cognition, $55,104-115$.

Luria, A. R. (1980). Higher Cortical Functions in Man. Nova Iorque: Basic Books.

Lane, R., Kaszniak, A., Ahern, G., \& Schwartz, G. (1997). Is alexithymia the emotional equivalent of blindsight? Biological Psychiatry, 42, 834-844.

Main, M., Kaplan, N., \& Cassidy, J. (1985). Security in infancy, childhood and adulthood: A move to the level of representation. Monographs of the Society for Research in Child Development, 50, 66-104.

Mota-Cardoso, R. (2001). Auto-regulação dos sistemas naturais. Revista Portuguesa de Psicossomática, 3, 39-96.

Pizzagalli, D., Regard, M., \& Lehmann, D. (1999). Rapid emotional face processing in the human right and left-brain hemispheres: An ERP study. NeuroReport, 10, 2691-2698.

Remper-Clower, N. L. (2007). Role of orbitofrontal cortex connections in emotion. Annals of the New York Academy of Sciences, 1121, 72-86.

Robinson, T. E., \& Kolb, B. (1999. Morphine alters the structure of neurons in nucleus accumbens and neocortex. Synapse, 33, 160-162.

Schore, A. (1994). Affect regulation and the origin of the self: The neurobiology of emotional development. Hillsdale, NJ: Lawrence Erlbaum.

Schore, A. (1997). Early organization of the nonlinear right brain and development of a predisposition to psychiatric disorders. Development and Psychopathology, 9, 595-631.

Schore, A. (2000). Attachment and the regulation of the right brain. Attachment \& Human Development, 1, 23-47.
Schore, A. (2001). Effects of a secure attachment, relationship on right brain development, affect regulation and infant mental health. Infant Mental Health Journal, 22, 7-66.

Starkstein, S. E., \& Robinson, R. G. (1997). Mechanism of disinhibition after brain lesions. Journal of Nervous and Mental Disease, 185, 108-114.

Stone, V. E., Baron-Cohen, S., \& Knight, R. T. (1998). Frontal lobe contributions to theory of mind. Journal of Cognitive Neuroscience, 10, 640-656.

Stronski, S. M., Ireland, M., Michaud, P., Narring F., \& Resnick, M. D. (2000). Protective correlates of stages in adolescent substance use: A Swiss National Study. Journal of Adolescent Health, 26, 420-427.

Subic-Wrana, C., Beutel, M., Garfield, D., \& Lane, R. (2011). Levels of emotional awareness: A model for conceptualizing and measuring emotion-centered structural change. International Journal of Psychoanalysis, 92, 289-310.

Trevarthen, C. (1993). The self-born in intersubjectivity: The psychology of an infant communicating. In U. Neisser (Ed.), The perceived self: Ecological and interpersonal sources of self-knowledge (pp. 121-173). New York: Cambridge University Press.

Verdejo-Garcia, A., \& Bechara, A. (2009). A somatic-marker theory of addiction. Neuropharmacology, 56, 48-62.

Winnicott, D. W. (1971). Playing and reality. London: Tavistock Publications.

Zald, D. H., \& Kim, S. W. (1996). Anatomy and function of the orbital frontal cortex, II: Function and relevance to obsessivecompulsive disorder. Journal of Neuropsychiatry, 8, 249-261.

Recebido em 02.01.2013

Primeira decisão editorial em 25.06.2014

Versão final em 30.07.2014

Aceito em 30.07.2014 